\title{
Macular and serum carotenoid concentrations in patients with malabsorption syndromes
}

\author{
Matthew S. Ward • Da You Zhao • Paul S. Bernstein
}

Received: 13 February 2008 / Accepted: 28 April 2008 / Published online: 13 June 2008

(C) The Author(s) 2008

\begin{abstract}
The carotenoids lutein and zeaxanthin are believed to protect the human macula by absorbing blue light and quenching free radicals. Intestinal malabsorption syndromes such as celiac and Crohn's disease are known to cause deficiencies of lipid-soluble nutrients. We hypothesized that subjects with nutrient malabsorption syndromes will demonstrate lower carotenoid levels in the macula and blood, and that these lower levels may correlate with early-onset maculopathy. Resonance Raman spectrographic (RRS) measurements of macular carotenoid levels were collected from subjects with and without a history of malabsorption syndromes. Carotenoids were extracted from serum and analyzed by high performance liquid chromatography (HPLC). Subjects with malabsorption $(n=22)$ had $37 \%$ lower levels of macular carotenoids on average versus controls $(n=25, P<0.001)$. Malabsorption was not associ-
\end{abstract}

Presented at the annual meeting of the Association for Research in Vision and Ophthalmology, Fort Lauderdale, Florida, May 2006.

Supported by NIH/NHLBI Grant 5 T35 HL07744-13 (Mr. Ward); Spectrotek, L.C., Salt Lake City, UT; National Eye Institute grant R01-EY-11600 (Dr. Bernstein); and a departmental grant from Research to Prevent Blindness, Inc, New York, NY. None of these funding sources were involved with the design and conduct of the study; collection, management, analysis, or interpretation of the data; or preparation, review, or approval of the manuscript. Dr. Bernstein had full access to all the data in the study and takes responsibility for the integrity of the data and the accuracy of the data analysis.

Financial/Proprietary Interests: Dr. Bernstein and the University of Utah hold a patent on the use of resonance Raman spectroscopy to measure macular carotenoid pigments in the human eye.

M. S. Ward • D. Y. Zhao • P. S. Bernstein $(\square)$

Department of Ophthalmology and Visual Sciences,

Moran Eye Center, University of Utah School of Medicine,

65 Mario Capecchi Drive,

Salt Lake City, Utah 84132, USA

e-mail: paul.bernstein@hsc.utah.edu ated with decreased serum carotenoid levels. Convincing signs of early maculopathy were not observed. We conclude that intestinal malabsorption results in lower macular carotenoid levels.

Keywords Macular carotenoids $\cdot$ Lutein $\cdot$ Zeaxanthin . Xanthophylls $\cdot$ Malabsorption

\section{Introduction}

Age-related macular degeneration (AMD) is the most common cause of irreversible blindness among elderly populations in the developed world [1]. A growing body of evidence suggests that AMD is in part a nutritional disease [2-9]. The nutritional basis for AMD is rooted in the theory that macula-specific dietary antioxidants quench reactive oxygen species and filter out high-energy blue light [9]. Studies have identified the fat-soluble carotenoids lutein and zeaxanthin as important components of the antioxidant protective system of the human macula [10].

Resonance Raman spectroscopy (RRS) is a novel technology for the objective, noninvasive measurement of macular carotenoids. We have shown previously that patients with AMD on average register 32\% lower concentrations of macular pigment versus controls as measured by RRS [11]. A review of this technology has been published previously [12].

Malabsorption syndromes comprise a group of disorders that result in diarrhea, steatorrhea, and/or malnutrition. Causes of malabsorption include celiac disease, inflammatory bowel disease (Crohn's disease and ulcerative colitis), cystic fibrosis, short bowel syndrome, pancreatitis, and infection. Studies suggest that malabsorption syndromes may lead to a state of chronic oxidative stress [13-15]. It is possible that such stress leads to increased incidence of 
oxidative diseases such as AMD in malabsorptive patients, but such a correlation has not yet been established.

Little has been written on the effect of nutrient malabsorption syndromes on the eye. Vitamin A (also fat soluble) dominates what literature there is, and a deficiency of this vitamin was most likely the first defined eye disease from malabsorption [16]. Of note, an early case report observed night-blindness and vitamin A deficiency after jejeunoileal bypass [17]. In 2000, a case report documented vitamin A deficiency in a patient with celiac disease, and a larger study demonstrated low serum carotene concentrations in patients with Crohn's disease [18, 19]. In an exploratory survey of patients with early-onset drusen (under 50 years old), reported in abstract form, the current authors observed that two of 11 participants had a concomitant malabsorption syndrome [20]. Given that even the earliest signs of maculopathy are found in fewer than $1 \%$ of the population under 50 years of age [21], these findings suggested a potential connection between nutrient deficiency states and overt maculopathy. We propose that patients with malabsorption syndromes may suffer an ocular deficiency of macular pigment. Macular pigment deficiency could lead to increased risk of maculopathy in younger populations, as it may accelerate the oxidative processes that normally become manifest only with advanced age. In the current study, we evaluate carotenoid concentrations in serum and maculae of patients with malabsorption syndromes using objective RRS technology. Additionally, we explore whether malabsorption may correlate with clinically observable early-onset maculopathy.

\section{Materials and methods}

\section{Experimental design}

This is a controlled cross-sectional study that measures macular and serum carotenoids as well as the presence of drusen in both study groups at a given point in time.

\section{Subjects}

This study was approved by the University of Utah Institutional Review Board, and all subjects signed institutionally approved informed consent forms that complied with the tenets of the Declaration of Helsinki. Control subjects were recruited from the clinic population of the Moran Eye Center at the University of Utah, School of Medicine. Subjects with malabsorption syndromes were recruited during the same time period by approved searches of the University of Utah Hospital electronic chart database for relevant ICD-9 codes. Patients with matching codes were contacted by mail and invited to participate on a voluntary basis. Additional subjects were referred by physicians in the Department of Gastroenterology at the same institution and from community support groups for malabsorption patients. Subjects with and without malabsorption syndromes participated in all study procedures. All subjects were under 58 years of age. A basic medical history including history of family eye disease, nutritional supplementation, and smoking was obtained from each subject. Visual acuity was assessed, and eyes were dilated using $2.5 \%$ phenylephrine $\mathrm{HCl}$ and $1 \%$ tropicamide ophthalmic solutions $15-20 \mathrm{~min}$ prior to measurements, and a dilated eye examined to exclude ocular pathology.

RRS measurements of human macular carotenoids

We used ocular resonance Raman spectroscopy to measure human macular carotenoid concentrations. Details of the instrumentation have been described elsewhere [11, 12]. For a typical measurement, a $0.5-\mathrm{mW}$ argon laser $(488 \mathrm{~nm})$ is directed as a 1-mm spot onto the subject's macula for $0.5 \mathrm{~s}$ through a series of lenses and filters after pupil dilation of $>6 \mathrm{~mm}$ has been achieved. The subjects self-align by superimposing the blue-green pilot laser beam on an array of collection fibers illuminated with a red light-emitting-diode (LED). Raman backscattered light is collected by a fiber optic collection bundle and analyzed by a Raman spectrometer. Raman carotenoid peaks identifying lutein and zeaxanthin are obtained at $1,159 \mathrm{~cm}^{-1}$ and $1,525 \mathrm{~cm}^{-1}$ with a good signal-tonoise ratio when the laser excitation beam is aimed at the fovea. The peak height at the carotenoid carbon-carbon double-bond stretch frequency of $1,525 \mathrm{~cm}^{-1}$ is quantified after subtraction of background fluorescence by Windowsbased computer software (Eye-C-Spec; Spectrotek, LC, Salt Lake City, Utah). The Raman signal intensity was expressed as Raman counts. Because subjects occasionally blink or misalign, the mean \pm standard deviation (SD) of the highest three of five measurements was used for statistical analysis $[11,12]$. Only one self-selected eye was dilated and measured by RRS for each subject.

\section{Serum carotenoid extraction}

Approximately $5 \mathrm{ml}$ of nonfasting venous blood was drawn from each subject into serum separating tubes (BD, San Jose, CA). Blood samples were centrifuged at 5,000 rpm for $5 \mathrm{~min}$ at $4{ }^{\circ} \mathrm{C}$, and then the serum was removed and immediately stored at $-75^{\circ} \mathrm{C}$ until analysis. Carotenoids (lipid soluble) were extracted from $100 \mu$ l of serum based on previously reported methods [22, 23]. Briefly, $100 \mu \mathrm{l}$ of serum was added to $200 \mu \mathrm{l} 0.1 \%$ butylated hydroxytoluene (BHT) in ethanol to precipitate the proteins, and then $500 \mu$ l ethyl acetate was added to extract the carotenoids. The sample was centrifuged at $2,000 \times g$ for $5 \mathrm{~min}$ at $4^{\circ} \mathrm{C}$, and the supernatant 
phase was collected. Then the sample was extracted with $500 \mu$ l ethyl acetate two more times and extracted with $500 \mu \mathrm{l}$ hexane once. The collected supernatants were combined and dried down under vacuum. The dried sample was dissolved in $1 \mathrm{ml}$ of $50 \%$ methanol and then extracted with $500 \mu \mathrm{l}$ of hexane three times. The collected supernatants were then dried down and re-dissolved in $100 \mu$ of running solvent prior to HPLC analysis. Fifty-microliter samples were injected onto the HPLC system. All procedures were performed under dim light and on ice if possible.

\section{Chromatographic system and HPLC analysis}

The analyses were performed on a HPLC system (Thermo Scientific, San Jose, CA) equipped with an auto-sampler, a UV/visible photodiode-array detector, and data analysis software. The separations were performed on a cyano column (Microsorb cyano $100 \AA$, $5 \mu \mathrm{m}, 25 \mathrm{~cm}$ length $\times 4.6 \mathrm{~mm}$ internal diameter, Rainin Instrument Co., Woburn, MA). The eluent consisted of a mixture of hexane (80\%), methylene chloride (19.4\%), methanol $(0.5 \%)$, and $N, N$-diisopropylethylamine $(0.1 \%)$. The monitoring wavelength was $450 \mathrm{~nm}$, and the column flow rate was $1.0 \mathrm{ml} / \mathrm{min}$. The column temperature was maintained at $20^{\circ} \mathrm{C}$. The running time was $20 \mathrm{~min}$ for each injection, and quantitation was by external reference standards of purified lutein and zeaxanthin. Sample analyses were performed in duplicate, and the mean was used for statistical analysis.

\section{Fundus examinations}

Fundus examinations were performed by retinal specialists at the Moran Eye Center. Photographs of visualized drusen were taken by the imaging team at the Moran Eye Center.

\section{Statistical analysis}

RRS and serum data were analyzed via linear regression plots. Normal and malabsorption populations were compared by analysis of variance (ANOVA), unpaired, two-tailed Student's $t$-tests, and Fisher exact tests as appropriate using SigmaStat software (Jandel Scientific, San Rafael, CA).

\section{Results}

Study subjects

A total of 22 patients with malabsorption syndromes participated in the study (18 with celiac disease and four with Crohn's disease). Of the celiac patients, 16 reported receiving a definitive diagnosis from a gastroenterologist. Two of the patients were self-diagnosed after improvement with a gluten-free diet. All Crohn's patients reported a definitive diagnosis from a gastroenterologist. Twenty five healthy subjects without a history of malabsorption participated as controls. Controls were volunteers from the greater Salt Lake City area and were excluded if they were older than 55 years and/or had a malabsorption syndrome. Two control subjects had unilateral blindness due to trauma or neurofibromatosis. One celiac patient had membranoproliferative glomerulonephritis (dense deposit disease) with associated widespread macular drusen. This subject was not included in the RRS macular pigment analysis due to possible confounding effects. No subjects had significant cataracts or other ocular pathology. Table 1 summarizes the study population characteristics showing that the two groups were well matched ( $P>0.05$ for all categories).

\section{RRS measurements of macular carotenoids}

On average, subjects with malabsorption syndromes showed 37\% lower concentrations of macular carotenoids compared to control subjects $(P=0.002)$ as measured by RRS (Fig. 1). In both control and malabsorption groups, concentrations of macular carotenoids declined with age, consistent with previously published findings in the Utah population using multiple macular pigment measurement methods [11, 12, 24, 25]. The decrease in carotenoid concentrations in malabsorption patients relative to controls was consistent across age because the regression lines are nearly parallel. There were no significant differences between patients with Crohn's and celiac disease when analyzed separately. The lowest macular pigment concentration in either group was found in a 27-year-old male with poorly controlled Crohn's disease. His RRS signal intensity was approximately one third the normal concentration.

Serum carotenoid analysis

Because detailed dietary histories were beyond the scope of this study, serum carotenoids were used as a biomarker of systemic carotenoid concentrations [26, 27]. No significant difference was seen in serum concentrations of lutein when comparing groups $(P>0.05)$, while serum zeaxanthin was significantly higher in the malabsorption group $(P=0.018$; Figs. 2 and 3). This difference was driven by the subjects with celiac disease who had $70 \%$ higher serum zeaxanthin concentrations than the normals $(P=0.003$; Fig. 4). Serum concentrations of both carotenoids increased with age in both groups $(P=0.007$ in normal group; $P=0.15$ in malabsorption patients). The lowest concentrations of both lutein $(18 \mathrm{ng} / \mathrm{ml})$ and zeaxanthin $(8 \mathrm{ng} / \mathrm{ml})$ measured in any subject occurred in the same 27-year-old subject with Crohn's disease. These concentrations were $12 \%$ and $20 \%$ of the normal average for lutein $(167 \mathrm{ng} / \mathrm{ml})$ and zeaxanthin 
Table 1 Patient population characteristics $^{\mathrm{a}}$

${ }^{a}$ There were no statistical differences between the normal and malabsorption populations for all categories $(P>0.05)$ ${ }^{\mathrm{b}} \mathrm{BMI}$ available on $60 \%$ of the malabsorption and normal populations

\begin{tabular}{llc}
\hline & Normal & Malabsorption syndromes \\
\hline Age (years; mean \pm SD) & $32 \pm 9.7$ & $38.7 \pm 11.4$ \\
Age range (years) & $22-50$ & $17-57$ \\
No. males & 11 & 6 \\
No. females & 14 & 16 \\
No. white persons & 21 & 22 \\
No. nonwhite persons & 4 & 0 \\
Current smokers & 1 & 3 \\
Past or nonsmokers & 24 & 19 \\
Eye color (blue; hazel/green; brown) & $13 ; 3 ; 9$ & $12 ; 7 ; 3$ \\
Dilated pupil size (mean \pm SD) & $8 \pm 1 \mathrm{~mm}$ & $8 \pm 1 \mathrm{~mm}$ \\
Median visual acuity & $20 / 20$ & $20 / 20$ \\
Visual acuity range & $20 / 15-20 / 60$ & $20 / 15-20 / 60$ \\
No. Crohn's disease & 0 & 4 \\
No. celiac disease & 0 & 18 \\
Body mass index $(\mathrm{BMI}$; mean \pm SD) & $27.1 \pm 6.4$ & $25.6 \pm 3.6$ \\
\end{tabular}
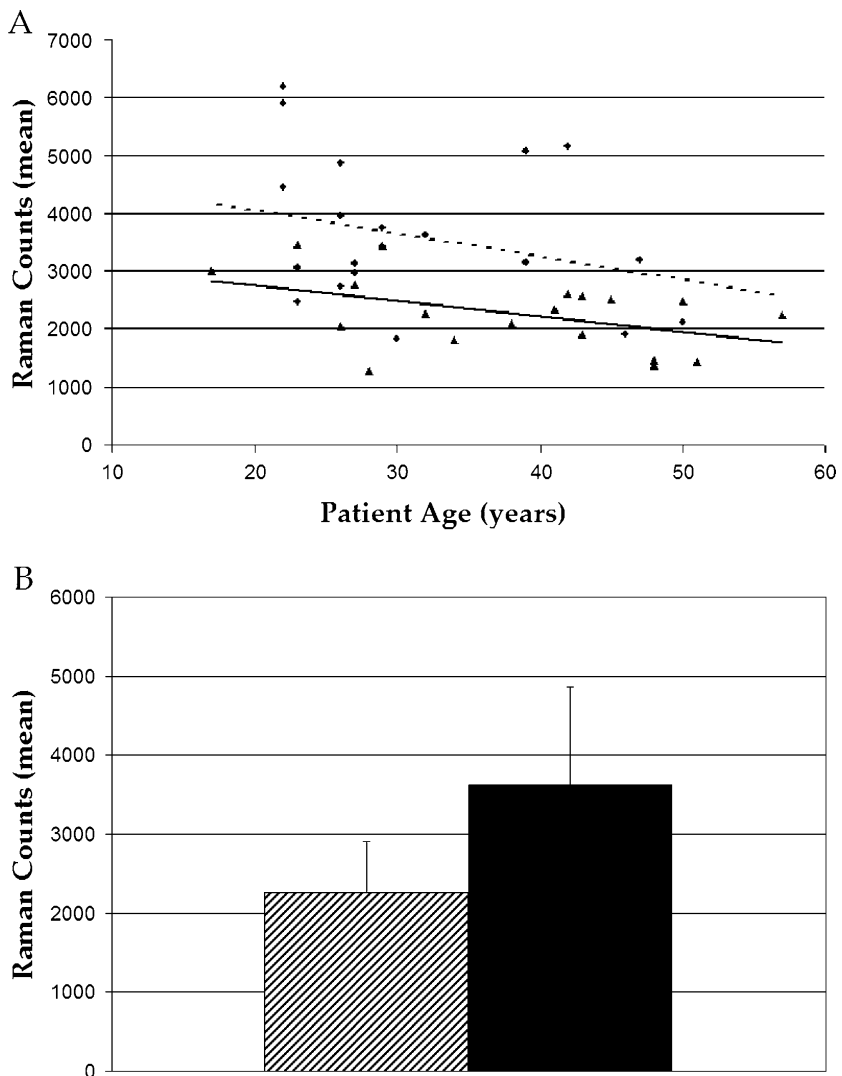

Fig. 1 RRS linear regression plot (a) and bar graph (b). The plot shows consistently lower Raman counts across age in malabsorption subjects (triangle points/solid line) versus normal subjects (circle points/dotted line; $P=0.002$ ). In both groups, the counts decline with age ( $P=0.20$ in normal subjects; $P=0.05$ in malabsorption patients). The bar graph compares Raman count averages in the both groups as a whole (malabsorption shaded, normal black). Malabsorption subjects registered $37 \%$ lower Raman counts on average compared to normals $(P<0.001)$
(59 $\mathrm{ng} / \mathrm{ml}$ ), respectively. His body mass index (BMI) was 21, which was also the lowest among subjects. No new significant differences were observed when data were stratified by race or sex.

\section{Fundus examinations}

Study subjects reported good ocular function in the tested eyes. A single, intermediate-sized soft druse was observed
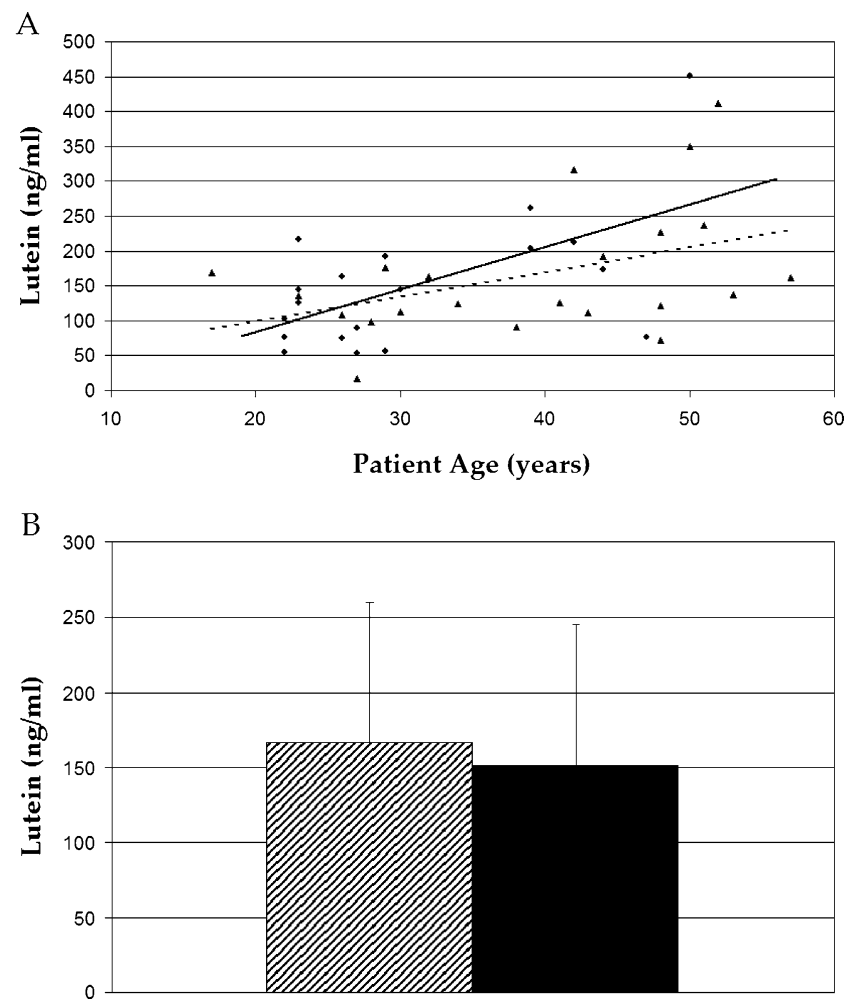

Fig. 2 Serum lutein regression plot (a) and bar graph (b) obtained by HPLC analysis. No difference in serum lutein concentrations were observed between malabsorption (triangle points/solid line, shaded bar) and normal subjects (circle points/dotted line, black bar, $P>0.05$ ) 

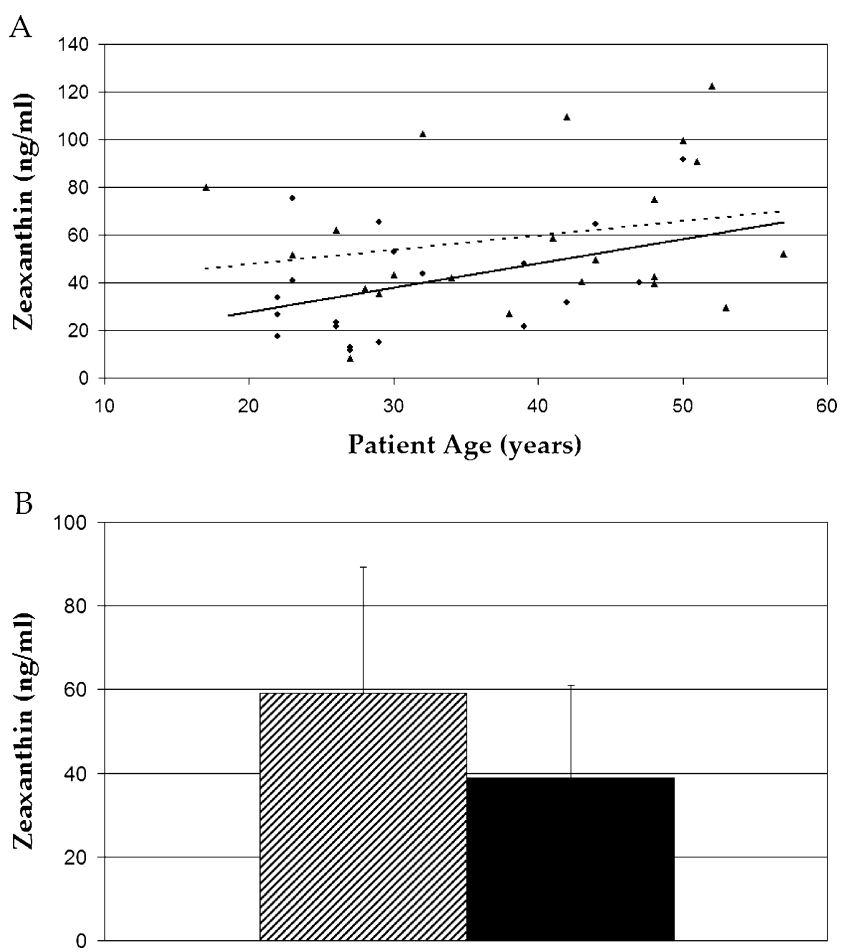

Fig. 3 Serum zeaxanthin regression plot (a) and bar graph (b) obtained by HPLC analysis. The bar graph indicates serum zeaxanthin is significantly higher in malabsorption subjects (triangle points/solid line, shaded bar) versus normals (circle points/dotted line, black bar, $P=0.018$ ) when grouped as a whole

in one 48 year-old subject with celiac disease. No other macular abnormalities were noted in malabsorption patients or controls with the exception of the celiac patient with membranoproliferative glomerulonephritis who had numerous macular drusen.

\section{Discussion}

Our finding that adults with intestinal malabsorption syndromes have decreased concentrations of macular pigment correlates well with a recent study that reported that patients with pancreatic insufficiency associated with cystic fibrosis registered lower concentrations of macular carotenoids in both serum and macula [28]. Despite the fact that neither study demonstrated convincing maculopathy at the time of examination, these patients certainly could be at risk for ongoing long-term macular oxidative stress that could predispose to AMD later in life.

Malabsorption in celiac disease results from mucosal villous blunting secondary to a T-cell mediated autoimmune response to ingested gluten (found in wheat, barley, rye, and oats) in the lamina propria of the small intestine [29]. Celiac disease is well controlled by a gluten-free diet in the majority of cases. Crohn's disease can present anywhere in the gastrointestinal tract from the mouth to the anus, and manifests in the form of recurrent, painful flares of accelerated autoimmune attack of the intestinal mucosal barrier [30]. The etiology is unknown, and the therapy is limited.

Unlike cystic fibrosis patients who have life-long, difficult to manage malabsorption syndromes, our malabsorption subjects generally had normal or even elevated serum concentrations of lutein and zeaxanthin. We propose some explanations for these initially unexpected findings. As mentioned above, celiac disease is widely known to be readily controllable. By eating a gluten-free diet, patients are able to achieve near complete remission of the disease, including mucosal villous recovery [31]. Whereas symptomatic relief usually occurs within two weeks, villous recovery is unpredictable and may take months to years [ibid]. Once recovery has taken place, there is no reason not to believe that a celiac patient would resume normal absorptive function. The majority of celiac subjects in the present study reported good control of their disease for over a year on a gluten-free diet. As such, we would expect their serum carotenoid concentrations to be in the normal range since serum concentrations typically equilibrate within weeks of any dietary change. Ideally, to validate our initial hypothesis of low serum carotenoid concentrations, we would have had to have enrolled only newly diagnosed celiac patients that had had minimal gluten-free diet control prior to carotenoid measurements, but these subjects are difficult to find. The higher than normal zeaxanthin concentrations in controlled celiac patients may be attributable to their increased consumption of corn which is a prominent part of a gluten-free diet and one of the richest known sources of zeaxanthin [32]. One study observed that subjects fed a corn-enriched diet for 15 weeks showed a $70 \%$ increase in serum zeaxanthin concentration [33].

Despite apparently normalized serum carotenoid concentrations in our malabsorption subjects, macular carotenoid

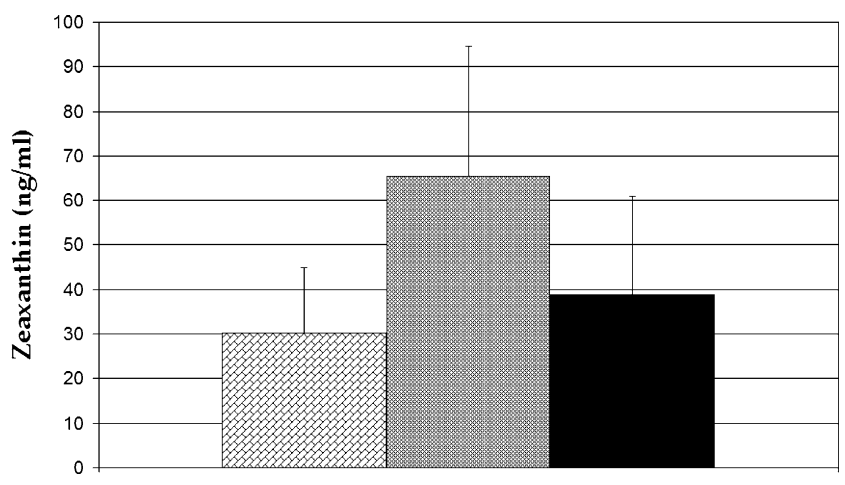

Fig. 4 Bar graph comparison of zeaxanthin concentrations. Crohn's patients are indicated by the diagonal brick-filled column, celiac patients by the shaded grey column, and normal subjects by the black column. On average, those with celiac disease had $70 \%$ higher serum zeaxanthin concentrations relative to normals $(P=0.003)$ 
concentrations were significantly below the age-matched normals. In normal human populations, dietary intake and serum lutein and zeaxanthin concentrations correlate only modestly with macular carotenoid concentrations [6], and evidence from studies in primates suggests that macular pigment concentrations in the serum and the macula at a given point in time are not well correlated [34]. Adult monkeys that were fed a life-long, xanthophyll-free diet retained the ability to absorb high quantities of these compounds following supplementation (as manifested by a rapid increase in serum concentrations of lutein and zeaxanthin); however, at 1 year post-supplementation, these same animals registered only half the amount of macular pigment in the macula as primates fed a life-long stock diet. It is not known whether macular concentrations ever normalized in the former group. Such a finding suggests the possibility that prolonged macular pigment deficiency may somehow affect the efficiency with which these pigments are transported to and concentrated in the macula. It is also possible that the concentration of lutein and zeaxanthin into macular tissue simply takes a long timepossibly years. A 2-year study of human macular pigment concentrations in the absence of dietary modification concluded that macular lutein and zeaxanthin concentrations were stable throughout the study period, despite seasonal fluctuations in serum concentrations [35]. Months-long drops in serum carotenoid concentrations yielded no significant change in macular concentrations. Considered together, these findings indicate that the movement of carotenoid into or out of the macula is most likely a slow process that is unaffected by acute fluctuations in serum concentrations. This makes sense from an evolutionary perspective in that the most advantageous biological system for protecting the macula would function despite the variable availability of carotenoidrich food. We therefore suggest that there is a lag between the resolution of serum carotenoid concentrations in patients with malabsorption syndromes (which may resolve within weeks of intestinal recovery) and macular carotenoid concentrations (which may require years to reaccumulate after years of deficiency). If these suppositions are true, they highlight the importance of early detection and monitoring of macular pigment deficiency, and they suggest that extended use of supplements may be required to reduce a patient's susceptibility to oxidative damage.

Serum lutein and zeaxanthin increased significantly with age in normal subjects. This finding contrasts with the agerelated decline in macular pigment noted in the same population. Adequate explanation for this observation is beyond the scope of this study. The molecular biology of carotenoid absorption, transport, and concentration with specificity to the macula is not well understood. One possible explanation is that an age-related breakdown in these pathways causes a decrease in the efficiency with which dietary carotenoids are taken up by the macula. If such is the case, a vegetable rich diet and/or carotenoid supplements may appreciably raise serum concentrations without a corresponding rise in the macula. Our population reported very little xanthophyll supplementation. Strict food histories including type and quantity of vegetable consumption were not obtained.

The markedly low concentrations of macular pigment detected across the board in one Crohn's patient indicate that this condition may produce a more severe, less controllable carotenoid deficiency than celiac disease. By subjective judgment and patient self-report, we deemed this patient to have the most poorly controlled malabsorptive disease. He reported poor Crohn's control on systemic steroids and frequent flares. He also smoked regularly $(1 / 2$ packs per day, ppd). Studies suggest that up to $85 \%$ of Crohn's patients admitted to the hospital for exacerbations have some degree of nutrient deficiency [36]. The percentage was $25 \%$ in the outpatient setting [37].

In summary, the present study offers further support for the potential for macular pigment deficiency in patients with malabsorption syndromes. We suggest that serum and macular concentrations of these pigments are unlikely to be correlated and may vary depending on the underlying malabsorption syndrome and the degree to which it is controlled. Additional study of a larger sample of malabsorptive patients is warranted to examine long-term risk of AMD. Such a study would be most effective in close collaboration with gastroenterologists to obtain data from subjects at the time of diagnosis and prior to any treatment measures.

Acknowledgments Matthew S. Ward participated in the experimental design, enrollment of subjects, the bulk of data collection and analysis, and writing of the manuscript. Da You Zhao assisted in collection and analysis of data, experimental consultation, and editing of the manuscript. Paul S. Bernstein is the principal investigator and participated in the experimental design, analysis of data, and writing and editing of the manuscript. Neither Dr. Ward nor Dr. Zhao has personal or commercial affiliations. Dr. Bernstein has commercial affiliation with Spectrotek, which manufactures the RRS technology.

Open Access This article is distributed under the terms of the Creative Commons Attribution Noncommercial License which permits any noncommercial use, distribution, and reproduction in any medium, provided the original author(s) and source are credited.

\section{References}

1. Klaver CC, et al. Age-specific prevalence and causes of blindness and visual impairment in an older population: the Rotterdam Study. Arch Ophthalmol 1998;116(5):653-8.

2. Landrum JT, Bone RA, Kilburn MD. The macular pigment: a possible role in protection from age-related macular degeneration. Adv Pharmacol 1997;38:537-56. 
3. Mares-Perlman JA, et al. Lutein and zeaxanthin in the diet and serum and their relation to age-related maculopathy in the third national health and nutrition examination survey. Am J Epidemiol 2001;153(5):424-32.

4. Mares-Perlman JA, et al. Association of zinc and antioxidant nutrients with age-related maculopathy. Arch Ophthalmol 1996;114(8):991-7.

5. Nolan JM, et al. Risk factors for age-related maculopathy are associated with a relative lack of macular pigment. Exp Eye Res 2007;84(1):61-74.

6. Nolan JM, et al. The relationships between macular pigment optical density and its constituent carotenoids in diet and serum. Invest Ophthalmol Vis Sci 2007;48(2):571-82.

7. Stringham JM, Hammond BR Jr. Dietary lutein and zeaxanthin: possible effects on visual function. Nutr Rev 2005;63(2):59-64.

8. Vu HT, et al. Does dietary lutein and zeaxanthin increase the risk of age related macular degeneration? The Melbourne Visual Impairment Project. Br J Ophthalmol 2006;90(3):389-90.

9. Winkler BS, et al. Oxidative damage and age-related macular degeneration. Mol Vis 1999;5:32.

10. Bone RA, et al. Analysis of the macular pigment by HPLC: retinal distribution and age study. Invest Ophthalmol Vis Sci 1988;29 (6):843-9.

11. Bernstein PS, et al. Resonance Raman measurement of macular carotenoids in normal subjects and in age-related macular degeneration patients. Ophthalmology 2002;109(10):1780-7.

12. Bernstein PS, et al. Resonance Raman measurement of macular carotenoids in the living human eye. Arch Biochem Biophys 2004;430(2):163-9.

13. Brown RK, Kelly FJ. Evidence for increased oxidative damage in patients with cystic fibrosis. Pediatr Res 1994;36(4):487-93.

14. Lepage G, et al. Supplementation with carotenoids corrects increased lipid peroxidation in children with cystic fibrosis. Am J Clin Nutr 1996;64(1):87-93.

15. Odetti P, et al. Oxidative stress in subjects affected by celiac disease. Free Radic Res 1998;29(1):17-24.

16. Wachtmeister L, et al. Attempts to define the minimal serum level of vitamin A required for normal visual function in a patient with severe fat malabsorption. Acta Ophthalmol (Copenh) 1988;66 (3):341-8.

17. Perlman I, et al. Night vision in a case of vitamin A deficiency due to malabsorption. Br J Ophthalmol 1983;67(1):37-42.

18. Alwitry A. Vitamin A deficiency in coeliac disease. $\mathrm{Br} \mathrm{J}$ Ophthalmol 2000;84(9):1079-80.

19. Imes S, et al. Vitamin A status in 137 patients with Crohn's disease. Digestion 1987;37(3):166-70.

20. Bernstein PS, Zhao DY, Wintch SW, Ermakov IV, McClane RW, Gellermann W. Resonance Raman spectroscopic measurement of macular carotenoid pigments in patients with early-onset macular drusen. Invest Ophthalmol Vis Sci. 2003;44:ARVO E-Abstract 1806.
21. Friedman DS, et al. Prevalence of age-related macular degeneration in the United States. Arch Ophthalmol 2004;122 (4):564-72.

22. Khachik F, et al. Identification, quantification, and relative concentrations of carotenoids and their metabolites in human milk and serum. Anal Chem 1997;69:1873-81.

23. Sowell AL, Huff HD, Gunter EW, Driskell WJ. Identification of cis-carotenoids in human sera analyzed by reversed-phase highperformance liquid chromatography with diode array detection. J Chromatogr Biomed Appl 1988;431:424-30.

24. Sharifzadeh M, Bernstein PS, Gellermann W. Nonmydriatic fluorescence-based quantitative imaging of human macular pigment distributions. J Opt Soc Am A Opt Image Sci Vis 2006;23 (10):2373-87.

25. Zhao DY, et al. Resonance Raman measurement of macular carotenoids in retinal, choroidal, and macular dystrophies. Arch Ophthalmol 2003;121(7):967-72.

26. Campbell DR, et al. Plasma carotenoids as biomarkers of vegetable and fruit intake. Cancer Epidemiol Biomarkers Prev 1994;3(6):493-500.

27. Gomez-Aracena J, et al. Vegetable consumption and carotenoids in plasma and adipose tissue in Malaga, Spain. Int J Vitam Nutr Res 2003;73(1):24-31.

28. Schupp C, et al. Lutein, zeaxanthin, macular pigment, and visual function in adult cystic fibrosis patients. Am J Clin Nutr 2004;79 (6): 1045-52.

29. Green $\mathrm{PH}$, et al. Mechanisms underlying celiac disease and its neurologic manifestations. Cell Mol Life Sci 2005;62(7-8):791-9.

30. Sartor RB. Mechanisms of disease: pathogenesis of Crohn's disease and ulcerative colitis. Nat Clin Pract Gastroenterol Hepatol 2006;3(7):390-407.

31. Farrell RJ, Kelly CP. Celiac sprue. N Engl J Med 2002;346 (3): $180-8$.

32. Panfili G, Fratianni A, Irano M. Improved normal-phase highperformance liquid chromatography procedure for the determination of carotenoids in cereals. J Agric Food Chem 2004;52 (21):6373-7.

33. Hammond BR Jr, et al. Dietary modification of human macular pigment density. Invest Ophthalmol Vis Sci 1997;38(9):1795801.

34. Neuringer M, et al. Nutritional manipulation of primate retinas, I: effects of lutein or zeaxanthin supplements on serum and macular pigment in xanthophyll-free rhesus monkeys. Invest Ophthalmol Vis Sci 2004;45(9):3234-43.

35. Nolan JM, et al. Monthly consistency of macular pigment optical density and serum concentrations of lutein and zeaxanthin. Curr Eye Res 2006;31(2):199-213.

36. Han PD, et al. Nutrition and inflammatory bowel disease. Gastroenterol Clin North Am 1999;28(2):423-43. ix.

37. Gassull MA, et al. Enteral nutrition in inflammatory bowel disease. Gut 1986;27(1):76-80. 